\title{
Inflammatory biomarkers in prognostic analysis for patients with glioma and the establishment of a nomogram
}

\author{
TONG YANG ${ }^{*}$, PING MAO* ${ }^{*}$ XIANHAI CHEN, XUAN NIU, GAOFENG XU, XIAOBIN BAI and WANFU XIE \\ Department of Neurosurgery, The First Affiliated Hospital of Xi'an Jiaotong University, Xi'an, Shaanxi 710061, P.R. China
}

Received June 14, 2017; Accepted December 19, 2018

DOI: $10.3892 / \mathrm{ol} .2018 .9870$

\begin{abstract}
Being biomarkers that reflect host nutritional and immune status, prognostic nutritional index (PNI) and neutrophil/lymphocyte ratio (NLR) have been identified to be independent prognostic factors in various malignancies. The aim of the present study was to determine the predictive value of these parameters for the prognosis of patients with glioma. Hematological and clinicopathological data were retrospectively analyzed from 128 patients with glioma who underwent brain tumor resection between January 2008 and December 2012. Receiver operating characteristic (ROC) analysis was used to determine the optimal cut-offs for PNI and NLR. Kaplan-Meier survival analysis, and univariate and multivariate analyses based on Cox proportional hazards regression model were used to determine whether NLR and PNI were associated with the prognosis of patients with glioma. R software was used to develop nomograms with all the independent prognostic factors included. Kaplan-Meier analysis followed by log-rank tests indicated that NLR $\geq 2.8$ and PNI $<45$ were significantly associated with decreased overall survival time. The subsequent multivariate analysis indicated that age $\geq 50$ years [hazard ratio (HR), 2.328; $95 \%$ confidence interval (CI), 1.386-3.908; $\mathrm{P}<0.001]$, high-grade glioma (HR, 3.088; 95\% CI, 1.893-5.037; P<0.001), gross total resection (HR, 0.606; 95\% CI, 0.380-0.965; $\mathrm{P}=0.035$ ) and NLR $\geq 2.8$ (HR, 2.037; 95\% CI, 1.264-3.281; P=0.003) were independent prognostic factors. The results of the present study indicated that high NLR was an independent risk factor for overall survival rates in patients with glioma, which indicated its value in improving the current prognostic model.
\end{abstract}

Correspondence to: Dr Wanfu Xie, Department of Neurosurgery, The First Affiliated Hospital of Xi'an Jiaotong University, 277 Yanta West Road, Xi'an, Shaanxi 710061, P.R. China

E-mail:wanfu67@aliyun.com

*Contributed equally

Key words: glioma, neutrophil/lymphocyte ratio, systemic inflammation, prognosis

\section{Introduction}

Glioma is the most common primary intracranial tumors, representing $81 \%$ of malignant brain tumors (1). Despite certain conclusive prognostic factors (e.g. extent of tumor resection, age at diagnosis and Karnofsky performance status) for glioma having been identified $(2,3)$, prognosis remains unsatisfactory, which indicates the involvement of other factors. Therefore, a more accurate prognostic indicator for patients with glioma is required.

Of note, emerging evidence has indicated that the systemic inflammation response is a key determinant of progression in cancer (4), which presents with alterations in circulating blood indicators (5). To date, several peripheral blood inflammation-based scores such as the neutrophil/lymphocyte ratio (NLR), platelet/lymphocyte ratio (PLR) have been validated as prognostic markers in various types of tumor (6-10). Furthermore, as a corrected parameter that reflects the host nutritional and immune condition, the prognostic nutritional index (PNI) has been identified to be associated with the progression of malignant tumors $(11,12)$. Generally, these combined scores are derived from established measures of full blood count, namely albumin, neutrophil, platelet and lymphocyte counts, reflecting a comprehensive individual state in aspects of systemic inflammation, nutritional and immune status.

However, the function of these blood-derived biomarkers in the prognosis of glioma is less well-investigated. As these parameters can be determined routinely in day-to-day clinical practice, it indicates promise for providing simpler and cheaper objective biomarkers for glioma prognosis. The aim of the present study was to identify the predictive value of these prognostic factors (i.e. NLR, PLR and PNI) in patients with glioma. To the best of our knowledge, for the first time, a predictive nomogram for prognosis in patients with glioma based on these biomarkers and the clinicopathological characteristics has been established.

\section{Materials and methods}

Ethics. The present study is a sequential study, with the patient information used acquired from a study previously published (13), which was approved by the Ethics Committee of The First Affiliated Hospital of Xi'an Jiaotong University (Xi'an, China; no. 2016-18). Written informed consent was obtained from all individual participants. 
Study population. The medical records of patients with newly diagnosed glioma who underwent brain tumor resection at The First Affiliated Hospital of Xi'an Jiaotong University between January 2008 and to December 2012 were retrospectively reviewed. Patients were included on the basis of eligibility criteria, which were: i) Diagnosis was confirmed by post-operative pathological examination; ii) full pre-operative laboratory data were available (i.e. serum albumin level, neutrophil, platelet and lymphocyte counts); and iii) no hematological system disorders, impaired liver function or primary tumor at other sites.

Data collection. Demographic information of patients, such as age at diagnosis, sex and clinical characteristics, including histopathological diagnosis of tumor [based on 2007 World Health Organization (WHO) brain tumor classification] (14), tumor location, extent of surgical resection (total gross resection or incomplete resection) were collected from the medical records. Tumor characteristics was assessed using pre-operative magnetic resonance imaging images. The extent of surgical resection was determined by the neurosurgeon during surgery.

Overall survival (OS) time was defined as the interval between the time of surgery and mortality. Patients were censored at the end of follow-up, if mortality had not occurred. The last follow-up was performed in October 2016.

NLR was calculated as neutrophil count $\left(10^{9}\right.$ cells $\left./ 1\right)$ divided by lymphocyte count $\left(10^{9}\right.$ cells/1); PLR was calculated as platelet count $\left(10^{9}\right.$ cells/l) divided by lymphocyte count $\left(10^{9}\right.$ cells/1); and PNI was calculated as albumin level $(\mathrm{g} / \mathrm{l})+$ $5 x$ total lymphocyte count $\left(10^{9}\right.$ cells/l) (15).

Statistical analyses. The optimal cut-off values for NLR and PNI were determined by plotting receiver operating characteristic (ROC) curves for OS prediction. Continuous variables are presented as the mean \pm standard deviation or median (range) on the basis of whether they fitted normal distribution or not (determined using a Kolmogorov-Smirnov test; $\mathrm{P}<0.05)$. Categorical data are presented as percentages. Survival analyses on categorical variables were performed using the Kaplan-Meier method and significant differences between groups were identified using the log-rank test. The associations of prognostic indicators with other categorized clinicopathological parameters were determined using the $\chi^{2}$ and unpaired t-test according to the type of variable. The Cox proportional hazards regression model was applied to perform univariate and multivariate analyses, and those variables that were identified to be statistically significant in the univariate analysis were analyzed further by multivariate analysis.

The rms package in R software was used to develop nomograms that predicted the probability of survival at 1, 3 and 5 years, taking into account all the possible prognostic factors. Harrell's concordance index (C-index) was used to evaluate the accuracy of predictions. The value ranged from 0.5 (agreement by chance) to 1 (perfect prediction) (16).

All statistical analyses were performed using SPSS (version 20.0; IBM Corp., Armonk, NY, USA) and R software (version 3.3.1; Institute for Statistics and Mathematics, Vienna, Austria). All statistical tests used in the present study were two-sided. $\mathrm{P}<0.05$ was considered to indicate a statistically significant difference.

\section{Results}

Baseline demographic and clinical measures. Following a thorough review of the medical records, a total of 128 patients (71 men and 57 women) were included in the present study. The median OS time was 19 months (range, 1-81 months). At the last follow-up, 79/128 patients had succumbed to various causes. The baseline demographic and clinical characteristics are presented in Table I.

Among all the patients, 67 cases were diagnosed as low-grade glioma (WHO G1 and G2) and 61 cases were diagnosed as high-grade glioma (WHO G3 and G4). The majority of the lesions were at one side of the cerebral hemisphere, with $55(43.0 \%)$ on the left and $60(46.9 \%)$ on the right. In addition, $63(49.2 \%)$ lesions were located in the frontal lobe, $34(26.6 \%)$ were in the temporal lobe, $13(10.2 \%)$ were in the occipital or parietal lobes, and the remainder of the lesions $(18,14.1 \%)$ were at the lateral ventricle or callosum.

Regarding treatment details, 77 (60.2\%) underwent a microsurgical total gross resection of the tumor, whereas 51 $(39.8 \%)$ had subtotal resection due to the inaccessible areas or eloquent brain regions in which the tumor was located. No patient in our cohort had palliative decompression surgery or tumor biopsy.

Associations of NLR and PNI with clinicopathological variables. As presented in Table I, the associations of NLR and PNI with clinicopathological variables were evaluated. In this cohort, it was identified that high NLR was significantly associated with advanced tumor grade $(\mathrm{P}<0.001)$. With regard to PNI, significant associations were identified with the extent of resection, age, the level of albumin and the level of platelet. Furthermore, it was identified that the level of PNI was negatively associated with NLR $(r=-0.203 ; \mathrm{P}<0.05)$.

Effect of NLR and PNI on OS time. Using the OS rate as the endpoint, ROC curves for NLR and PNI were plotted (Fig. 1A and B).

For NLR [area under the curve (AUC), 0.639; 95\% confidence interval (CI), 0.542-0.733; $\mathrm{P}=0.009$ ], a cut-off of 2.8 led to the maximal Youden value in ROC curve analysis. Patients were subsequently categorized into two groups according to this cut-off value, with the high NLR group at $\geq 2.8$ and the low NLR group at $<2.8$. Kaplan-Meier survival analysis and log-rank tests indicated a significant difference in the median OS time between the high NLR group and low NLR group $(22.78 \pm 3.61$ vs. $48.31 \pm 4.01$ months; $\mathrm{P}<0.001)$, which indicated a marked association between high NLR and decreased OS time (Fig. 2A).

Accordingly, the prognostic effect of PNI was evaluated. Using ROC curves analysis, the AUC for PNI was 0.618 (95\% CI, 0.520-0.716; $\mathrm{P}=0.025$ ). The optimal cut-off value was further determined as 45.0. The median OS time was $43.55 \pm 3.44$ months in the high PNI group and $13.33 \pm 2.73$ months in the low PNI group $(\mathrm{P}<0.001)$, which indicated that low PNI was significantly associated with decreased OS time (Fig. 2B). 
Table I. Baseline characteristics of 128 patients with glioma and their associations with NLR and PNI.

\begin{tabular}{|c|c|c|c|c|c|c|c|}
\hline Variable & $\begin{array}{l}\text { All patients } \\
(\mathrm{n}=128)\end{array}$ & $\begin{array}{c}\mathrm{NLR}<2.8 \\
(\mathrm{n}=72)\end{array}$ & $\begin{array}{c}\mathrm{NLR} \geq 2.8 \\
\quad(\mathrm{n}=56)\end{array}$ & P-value & $\begin{array}{c}\mathrm{PNI}<45 \\
(\mathrm{n}=24)\end{array}$ & $\begin{array}{l}\mathrm{PNI} \geq 45 \\
(\mathrm{n}=104)\end{array}$ & P-value \\
\hline Sex, n (\%) & & & & 0.858 & & & 0.260 \\
\hline Female & $57(44.5)$ & $33(45.8)$ & $24(42.9)$ & & $8(33.3)$ & $49(47.1)$ & \\
\hline Male & $71(55.5)$ & $39(54.2)$ & $32(57.1)$ & & $16(66.7)$ & $55(52.9 \%)$ & \\
\hline Histology, n (\%) & & & & $<0.001$ & & & 0.266 \\
\hline LGG & $67(52.3)$ & $48(66.7)$ & $19(33.9)$ & & $10(41.7)$ & $57(54.8)$ & \\
\hline HGG & $61(47.7)$ & $24(33.3)$ & $37(66.1)$ & & $14(58.3)$ & $47(77.0)$ & \\
\hline Lateral, n (\%) & & & & 0.759 & & & 0.587 \\
\hline Right & $60(46.9)$ & $32(44.4)$ & $28(50.0)$ & & $9(37.5)$ & $51(49.0)$ & \\
\hline Left & $55(43.0)$ & $33(45.8)$ & $22(39.3)$ & & $12(50.0)$ & $43(41.3)$ & \\
\hline Bilateral & $13(10.2)$ & $7(9.7)$ & $6(10.7)$ & & $3(12.5)$ & $10(9.6)$ & \\
\hline Tumor location, n (\%) & & & & 0.773 & & & 0.268 \\
\hline Frontal lobe & $63(49.2)$ & $38(52.8)$ & $25(44.6)$ & & $10(41.7)$ & $53(51.0)$ & \\
\hline Temporal lobe & $34(26.6)$ & $18(25.0)$ & $16(28.6)$ & & $10(41.7)$ & $24(23.1)$ & \\
\hline Occipital/parietal lobe & $13(10.2)$ & $6(8.3)$ & $7(12.5)$ & & $1(4.2)$ & $12(11.5)$ & \\
\hline Other & $18(14.1)$ & $10(13.9)$ & $8(14.3)$ & & $3(12.5)$ & $15(14.4)$ & \\
\hline Extent of surgery, $n(\%)$ & & & & 0.857 & & & 0.005 \\
\hline Gross total & $77(60.2)$ & $44(61.1)$ & $33(58.9)$ & & $8(33.3)$ & $69(66.3)$ & \\
\hline Subtotal & $51(39.8)$ & $28(38.9)$ & $23(41.1)$ & & $16(66.7)$ & $35(33.7)$ & \\
\hline Age, years & $47.84 \pm 13.958$ & $46.22 \pm 13.67$ & $49.93 \pm 14.42$ & 0.137 & $56.79 \pm 10.43$ & $45.78 \pm 13.89$ & $<0.001$ \\
\hline Albumin (g/l) & $41.1 \pm 3.7$ & $41.13 \pm 3.53$ & $41.1 \pm 3.9$ & 0.966 & $37.3 \pm 3.2$ & $41.99 \pm 3.2$ & $<0.001$ \\
\hline Platelets $\left(\times 10^{9} / \mathrm{l}\right)$ & $176.8 \pm 61.9$ & $175.29 \pm 51.4$ & $178.82 \pm 73.76$ & 0.750 & $148.13 \pm 47.97$ & $183.46 \pm 63.07$ & 0.011 \\
\hline
\end{tabular}

P-values in italics are statistically significant. Age, albumin level and platelet count are presented as mean \pm standard deviation. NLR, neutrophil/lymphocyte ratio; PNI, prognostic nutritional index; LGG, low-grade glioma; HGG, high-grade glioma.

With regard to the remaining parameters, the cut-off values were determined by respective median or relative diagnosis criteria, as presented in Table II.

Additionally, Cox univariate analysis was used to further investigate the predictive value of NLR, PLR, PNI and other clinicopathological parameters. The results indicated that age, histology, extent of resection, level of albumin, NLR and PNI were significantly associated with OS. Furthermore, multivariate analysis indicated that age $\geq 50$ years [hazard ratio (HR), 2.328; 95\% CI, 1.386-3.908; $\mathrm{P}<0.001$ ], high-grade glioma (HR, 3.088; 95\% CI, 1.893-5.037; $\mathrm{P}<0.001)$, gross total resection (HR, 0.606; 95\% CI, 0.380-0.965; $\mathrm{P}=0.035$ ) and $\mathrm{NLR} \geq 2.8$ (HR, 2.037; 95\% CI, 1.264-3.281; $\mathrm{P}=0.003$ ) were independent prognostic factors for poor prognosis (Table II).

Predictive nomogram for prognosis in patients with glioma. A nomogram to predict the survival of patients with glioma following surgical resection is presented in Fig. 3A. With all the independent prognostic indicators included, the nomogram is able to provide an approximate prediction of the survival rate at 1, 3 and 5 years for patients with glioma. In the nomogram, all the indicators were scored as a number. For instance, if the patient had a high-grade glioma, the patient was attributed with 10 points. The total points of the patient were corresponding to a certain survival rate at 1, 3 and 5 years, and a greater number of points indicates a worse prognosis.
The nomogram exhibited a good accuracy for predicting the survival rate of patients with glioma, with a c-index of 0.750 . The value became 0.727 if NLR was not included in the nomogram. The internal calibration plots of the nomogram predicting 1-, 3- and 5-year survival revealed good agreement with the ideal model (Fig. 3B-D).

\section{Discussion}

Currently, maximal safe resection and adjunctive chemo/radiotherapy are considered to be the most effective treatment modalities for glioma. Since patients who have undergone the same treatment may have significant variation in their prognosis, a more accurate prognostic model is required to guide post-operative counseling and post-operative adjuvant treatment.

Notably, evidence has indicated the relevance between deteriorated nutritional and immunological condition with progress of cancer (17). As biomarkers of systemic inflammation, NLR and PLR have been identified as independent prognostic factors in a wide range of malignancies (6-8). In the present study, it was identified that NLR $\geq 2.8$ was significantly associated with decreased OS rates as an independent risk factor, which is consistent with the results of previous studies $(18,19)$. There are several potential mechanisms for how systemic inflammation is involved in tumor progression 

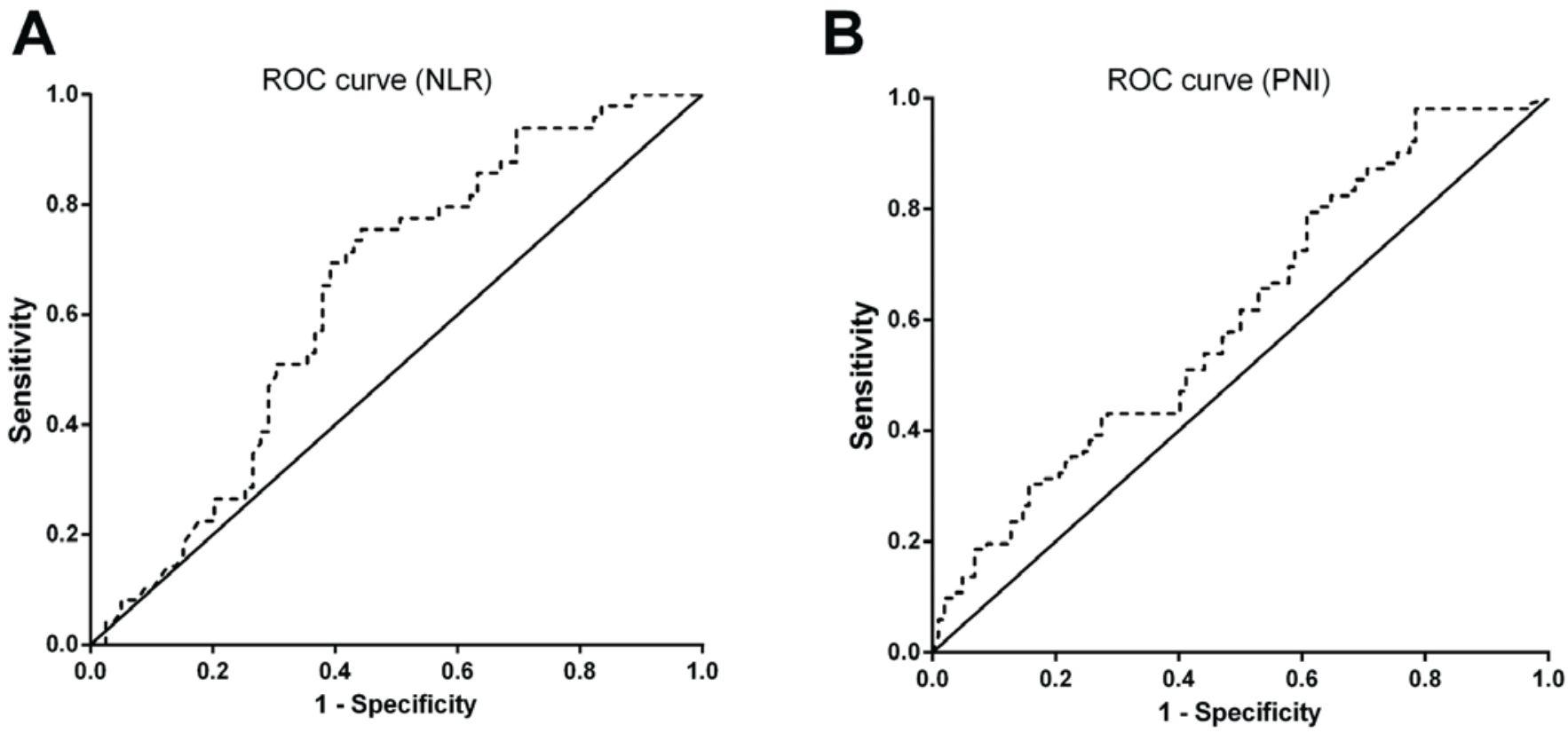

Figure 1. ROC curves analysis for optimal cut-offs for (A) NLR and (B) PNI. The optimal cut-offs for NLR and PNI are 2.8 and 45, respectively. ROC, receiver operating characteristic; NLR, neutrophil/lymphocyte ratio; PNI, prognostic nutritional index.
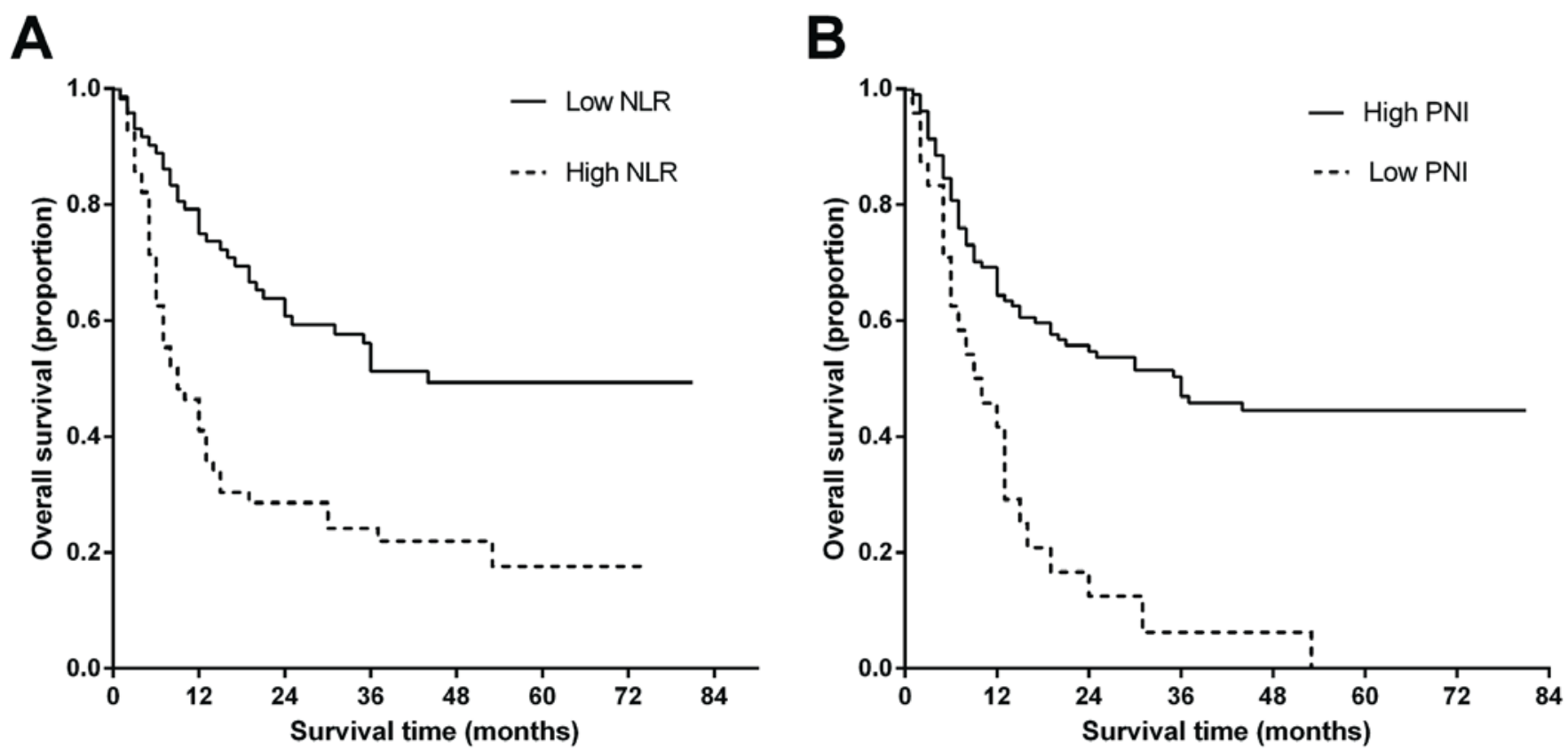

Figure 2. Overall Kaplan-Meier survival curves stratified by (A) NLR or (B) PNI. NLR, neutrophil/lymphocyte ratio; PNI, prognostic nutritional index.

and recurrence. One is that the inflammation response deteriorated the cellular immune response to malignancy by depleting lymphocytes (20). Secondly, the relatively increased neutrophil level was deemed to contain and promote the release of inflammatory mediators and vascular endothelial growth factor, leading to the promotion of angiogenesis, DNA damage and genetic instability which have been widely accepted to be associated with tumor proliferation and metastasis (21-23).

PNI, a corrected indicator for the nutritional and immunological condition of patients, was proposed a few decades ago. In the early days, PNI was used to assess the risk of post-operative complications, particularly the surgery for gastrointestinal malignancy (24). With the predictive value of PNI being validated in other tumors (25), it began to be accepted that this factor was not determined by the digestive or liver function alone. A previous study revealed that the inflammatory environment alteration induced by cancer cells may be the reason for the deterioration of the nutritional condition of patients (26). In the present study, a significant difference in OS time between the patients with high PNI and low PNI was identified. However, the multivariate analysis indicated that the PNI was not an independent prognostic factor. The reason for this may be that NLR weakened the test power of PNI, as NLR is a relatively more direct indicator for systemic inflammation compared with PNI. 
Table II. Univariate and multivariate analyses for overall survival time of patients with glioma.

\begin{tabular}{|c|c|c|c|c|}
\hline \multirow[b]{2}{*}{ Variable } & \multicolumn{2}{|c|}{ Univariate } & \multicolumn{2}{|c|}{ Multivariate } \\
\hline & HR $(95 \%$ CI) & P-value & $\mathrm{HR}(95 \% \mathrm{CI})$ & P-value \\
\hline \multicolumn{5}{|l|}{ Sex } \\
\hline Female & 1.000 & & & \\
\hline Male & $0.910(0.583-1.419)$ & 0.676 & & \\
\hline \multicolumn{5}{|l|}{ Age, years } \\
\hline$<50$ & 1.000 & & & \\
\hline$\geq 50$ & $3.166(1.973-5.081)$ & $<0.001$ & $2.328(1.386-3.908)$ & 0.001 \\
\hline \multicolumn{5}{|l|}{ Tumor location } \\
\hline Frontal lobe & $1.303(0.629-2.698)$ & 0.476 & & \\
\hline Temporal lobe & $1.942(0.904-4.171)$ & 0.089 & & \\
\hline Occipital/parietal lobe & $1.245(0.463-3.347)$ & 0.664 & & \\
\hline Other & 1.000 & & & \\
\hline \multicolumn{5}{|l|}{ Lateral } \\
\hline Right & $1.109(0.519-2.372)$ & 0.790 & & \\
\hline Left & $0.853(0.392-1.858)$ & 0.689 & & \\
\hline Bilateral & 1.000 & & & \\
\hline \multicolumn{5}{|l|}{ Histology } \\
\hline LGG & 1.000 & & & \\
\hline HGG & $3.675(2.284-5.912)$ & $<0.001$ & $3.088(1.893-5.037)$ & $<0.001$ \\
\hline \multicolumn{5}{|l|}{ Extent of resection } \\
\hline Gross total & $0.557(0.357-0.869)$ & 0.010 & $0.606(0.380-0.965)$ & 0.035 \\
\hline Subtotal & 1.000 & & & \\
\hline \multicolumn{5}{|l|}{ Albumin, g/l } \\
\hline$\geq 35$ & 1.000 & & & \\
\hline$<35$ & $0.313(0.124-0.787)$ & 0.014 & $0.675(0.243-1.880)$ & 0.452 \\
\hline \multicolumn{5}{|l|}{ Platelets, x 109/1 } \\
\hline$<350$ & 1.000 & & & \\
\hline$\geq 350$ & $3.279(0.449-23.956)$ & 0.242 & & \\
\hline \multicolumn{5}{|l|}{ NLR } \\
\hline$<2.8$ & 1.000 & & & \\
\hline$\geq 2.8$ & $2.525(1.611-3.957)$ & $<0.001$ & $2.037(1.264-3.281)$ & 0.003 \\
\hline \multicolumn{5}{|l|}{ PLR } \\
\hline$\geq 100$ & 1.000 & & & \\
\hline$<100$ & $1.348(0.850-2.138)$ & 0.205 & & \\
\hline \multicolumn{5}{|l|}{ PNI } \\
\hline$<45$ & 1.000 & & & \\
\hline$\geq 45$ & $0.346(0.210-0.569)$ & $<0.001$ & $0.716(0.400-1.280)$ & 0.260 \\
\hline
\end{tabular}

As a novel prediction method for prognosis, nomograms have been widely used in oncology (27). In the present study, age at diagnosis, tumor character, extent of resection and the inflammatory condition of the patients were taken into account when the predictive nomogram was established, providing an improved prediction of the individual prognosis following surgery in a more intuitive way and provided guidance for clinicians to decide whether adjuvant treatment is required.
Additionally, to the best of our knowledge, the present study is the first to use a nomogram to evaluate the prognostic value of NLR for patients with glioma.

The present study has a number of limitations. First, the sample size is relatively small and all the subjects included were from a single institution. Secondly, owing to the retrospective nature of the study, information bias and selection bias are essentially inevitable. Therefore, prospective studies 
A

Points

Age

Histology

Extent of resection

NLR

Total points

1-year survival

3-year survival

5-year survival

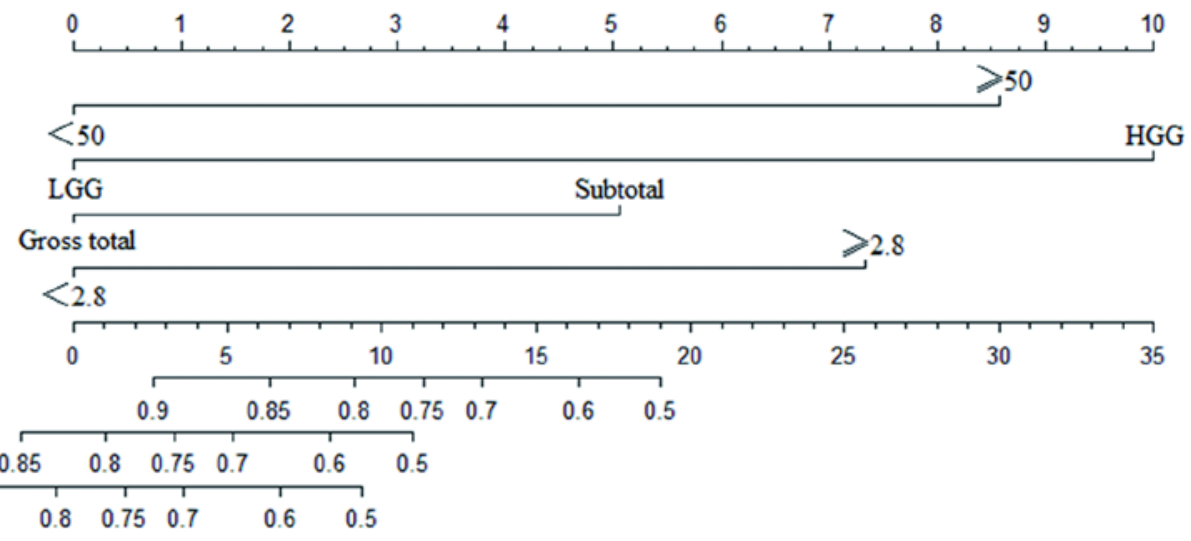
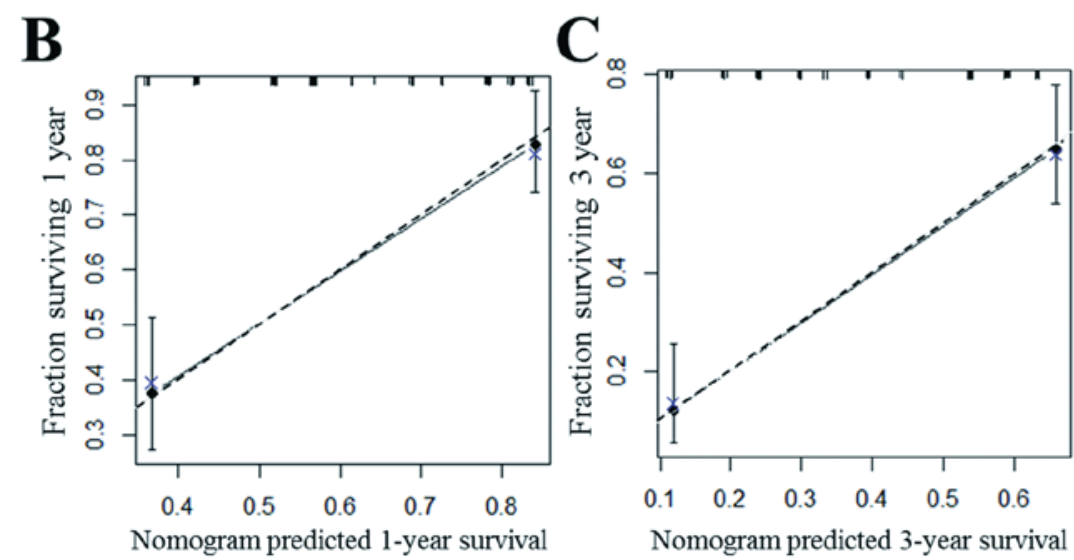

D

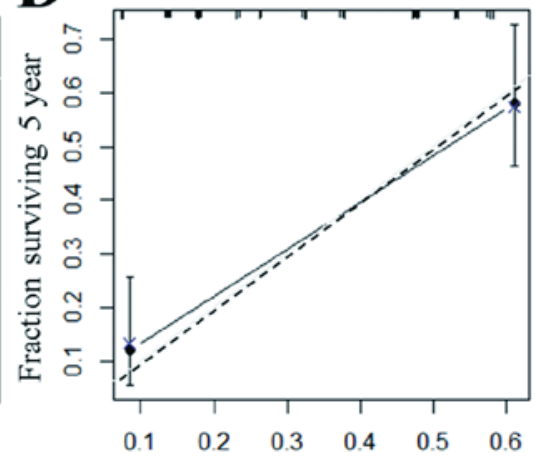

Nomogram predicted 5-year survival

Figure 3. (A) Nomogram for predicting the survival rate at 1,3 and 5 years after initial treatment. Internal calibration plots of the nomogram for (B) 1-year, (C) 3-year and (D) 5-year survival. NLR, neutrophil/lymphocyte ratio; LGG, low-grade glioma; HGG, high-grade glioma.

involving a large sample size and thorough follow-up are required to investigate further the association between inflammatory biomarkers and prognosis in patients with glioma.

In conclusion, the present study indicated that high NLR was an independent risk factor for OS time in patients with glioma, which indicated its value for improving the current prognostic model.

\section{Acknowledgements}

Not applicable.

\section{Funding}

The present study was funded by Natural Science Foundation of Shaanxi Province (grant no., 2015JM8462) and National Natural Science Foundation of China (grant no., 81602207).

\section{Availability of data and materials}

The datasets used and analyzed during the current study are available from the corresponding author on reasonable request.

\section{Authors' contributions}

TY analyzed data and drafted the manuscript. PM participated in data interpretation and manuscript revision. XC participated in data acquisition and analysis. $\mathrm{XN}$ participated in data analysis and interpretation. GX and XB participated in study concept and data acquisition. WX participated in study concept and design. All authors read and approved the final manuscript.

\section{Ethics approval and consent to participate}

The present study is a sequential study, with the patient information used acquired from a study previously published (13), which was approved by the Ethics Committee of The First Affiliated Hospital of Xi'an Jiaotong University (Xi'an, China; no. 2016-18). Written informed consent was obtained from all individual participants.

\section{Patient consent for publication}

Consent was obtained from all patients for the publication of the present study.

\section{Competing interests}

The authors declare that they have no competing interests.

\section{References}

1. Ostrom QT, Bauchet L, Davis FG, Deltour I, Fisher JL, Langer CE, Pekmezci M, Schwartzbaum JA, Turner MC, Walsh KM, et al: The epidemiology of glioma in adults: A 'state of the science' review. Neuro Oncol 16: 896-913, 2014. 
2. Gorlia T, van den Bent MJ, Hegi ME, Mirimanoff RO, Weller M, Cairncross JG, Eisenhauer E, Belanger K, Brandes AA, Allgeier A, et al: Nomograms for predicting survival of patients with newly diagnosed glioblastoma: Prognostic factor analysis of EORTC and NCIC trial 26981-22981/CE. 3. Lancet Oncol 9: 29-38, 2008

3. Liang TH, Kuo SH, Wang CW, Chen WY, Hsu CY, Lai SF, Tseng HM, You SL, Chen CM and Tseng WY: Adverse prognosis and distinct progression patterns after concurrent chemoradiotherapy for glioblastoma with synchronous subventricular zone and corpus callosum invasion. Radiother Oncol 118: 16-23, 2016

4. Mantovani A, Allavena P, Sica A and Balkwill F: Cancer-related inflammation. Nature 454: 436-444, 2008.

5. Gabay C and Kushner I: Acute-phase proteins and other systemic responses to inflammation. N Engl J Med 340: 448-454, 1999.

6. Templeton AJ, Ace O, McNamara MG, Al-Mubarak M, Vera-Badillo FE, Hermanns T, Seruga B, Ocaña A, Tannock IF and Amir E: Prognostic role of platelet to lymphocyte ratio in solid tumors: A systematic review and meta-analysis. Cancer Epidemiol Prevention Biomarkers Prev 23: 1204-1212, 2014.

7. Guthrie GJ, Charles KA, Roxburgh CS, Horgan PG, McMillan DC and Clarke SJ: The systemic inflammation-based neutrophil-lymphocyte ratio: Experience in patients with cancer. Crit Rev Oncol Hematol 88: 218-230, 2013.

8. Yang HB, Xing M, Ma LN, Feng LX and Yu Z: Prognostic significance of neutrophil-lymphocyteratio/platelet-lymphocyteratioin lung cancers: A meta-analysis. Oncotarget 7: 76769-76778, 2016

9. Kijima T, Arigami T, Uchikado Y, Uenosono Y, Kita Y, Owaki T, Mori S, Kurahara H, Kijima Y, Okumura H, et al: Combined fibrinogen and neutrophil-lymphocyte ratio as a prognostic marker of advanced esophageal squamous cell carcinoma. Cancer Sci 108: 193-199, 2017.

10. Suzuki R, Takagi T, Hikichi T, Konno N, Sugimoto M, Watanabe KO, Nakamura J, Waragai Y, Kikuchi H, Takasumi M, et al: Derived neutrophil/lymphocyte ratio predicts gemcitabine therapy outcome in unresectable pancreatic cancer. Oncol Lett 11: 3441-3445, 2016.

11. Yang Y, Gao P, Chen X, Song Y, Shi J, Zhao J, Sun J, Xu Y and Wang Z: Prognostic significance of preoperative prognostic nutritional index in colorectal cancer: Results from a retrospective cohort study and a meta-analysis. Oncotarget 7: 58543-58552, 2016.

12. Yang Z, Zhang B, Hou L, Xie Y and Cao X: Pre-operative prognostic nutritional index predicts the outcomes for triple-negative breast cancer. Tumor Biol 35: 12165-12171, 2014.

13. Wang J, Yang T, Xu G, Liu H, Ren C, Xie W and Wang M: Cyclin-dependent kinase 2 promotes tumor proliferation and induces radio resistance in glioblastoma. Transl Oncol 9 548-556, 2016

14. Louis DN, Ohgaki H, Wiestler OD, Cavenee WK, Burger PC,Jouvet A, Scheithauer BW and Kleihues P: The 2007 WHO classification of tumours of the central nervous system. Acta Neuropathol 114: 97-109, 2007.
15. Onodera T, Goseki N and Kosaki G: Prognostic nutritional index in gastrointestinal surgery of malnourished cancer patients. Nihon Geka Gakkai Zasshi 85: 1001-1005, 1984 (In Japanese)

16. Harrell FE Jr, Lee KL and Mark DB: Multivariable prognostic models: Issues in developing models, evaluating assumptions and adequacy, and measuring and reducing errors. Stat Med 15: 361-387, 1996.

17. Crusz SM and Balkwill FR: Inflammation and cancer: Advances and new agents. Nat Rev Clin Oncol 12: 584-596, 2015.

18. Bambury RM, Teo MY, Power DG, Yusuf A, Murray S, Battley JE, Drake C, O'Dea P, Bermingham N, Keohane C, et al: The association of pre-treatment neutrophil to lymphocyte ratio with overall survival in patients with glioblastoma multiforme. J Neurooncol 114: 149-154, 2013.

19. Auezova R, Ryskeldiev N, Doskaliyev A, Kuanyshev Y, Zhetpisbaev B, Aldiyarova N, Ivanova N, Akshulakov S and Auezova L: Association of preoperative levels of selected blood inflammatory markers with prognosis in gliomas. OncoTargets Ther 9: 6111-6117, 2016.

20. Denkert C, Loibl S, Noske A, Roller M, Müller BM, Komor M, Budczies J, Darb-Esfahani S, Kronenwett R, Hanusch C, et al: Tumor-associated lymphocytes as an independent predictor of response to neoadjuvant chemotherapy in breast cancer. J Clin Oncol 28: 105-113, 2010.

21. Grivennikov SI, Greten FR and Karin M: Immunity, inflammation, and cancer. Cell 140: 883-899, 2010.

22. Jablonska J, Leschner S, Westphal K, Lienenklaus S and Weiss S: Neutrophils responsive to endogenous IFN-beta regulate tumor angiogenesis and growth in a mouse tumor model. J Clin Invest 120: 1151-1164, 2010.

23. Lord BI, Bronchud MH, Owens S, Chang J, Howell A, Souza L and Dexter TM: The kinetics of human granulopoiesis following treatment with granulocyte colony-stimulating factor in vivo. Proc Natl Acad Sci USA 86: 9499-9503, 1989.

24. Buzby GP, Mullen JL, Matthews DC, Hobbs CL and Rosato EF: Prognostic nutritional index in gastrointestinal surgery. Am J Surg 139: 160-167, 1980.

25. Ma W, Zhang P, Qi J, Gu L, Zang M, Yao H, Shi X, Wang C and Jiang Y: Prognostic value of platelet to lymphocyte ratio in hepatocellular carcinoma: A meta-analysis. Sci Rep 6: 35378, 2016.

26. Lo HC, Tsao LY, Hsu WY, Chen HN, Yu WK and Chi CY: Relation of cord serum levels of growth hormone, insulin-like growth factors, insulin-like growth factor binding proteins, leptin, and interleukin- 6 with birth weight, birth length, and head circumference in term and preterm neonates. Nutrition 18: 604-608, 2002

27. Balachandran VP, Gonen M, Smith JJ and DeMatteo RP: Nomograms in oncology: More than meets the eye. Lancet Oncol 16: e173-e180, 2015. 